\title{
The pathophysiology of letter-by-letter reading
}

\author{
Laurent Cohen $^{\mathrm{a}, \mathrm{b}, *}$, Carole Henry ${ }^{\mathrm{a}, \mathrm{b}}$, Stanislas Dehaene $^{\mathrm{b}}$, Olivier Martinaud ${ }^{\mathrm{a}, \mathrm{b}}$, \\ Stéphane Lehéricy ${ }^{\mathrm{c}}$, Cathy Lemer ${ }^{\mathrm{b}}$, Sophie Ferrieux ${ }^{\mathrm{a}}$ \\ a Institut de Neurologie, Hôpital de la Salpêtrière, 47/83 Bd de l'Hôpital, 75651 Paris CEDEX 13, France \\ b INSERM U562, Service Hospitalier Frédéric Joliot, CEA/DSV, Orsay, France \\ c Service de Neuroradiologie, Hôpital de la Salpêtrière, AP-HP, Paris, France
}

Received 24 March 2004; received in revised form 28 April 2004; accepted 30 April 2004

\begin{abstract}
Pure alexia is a frequent and incapacitating consequence of left occipitotemporal lesions. It is thought to result from the disruption or the disconnection of the visual word form area (VWFA), a region reproducibly located within the left occipito-temporal sulcus, and encoding the abstract identity of strings of visual letters. Alexic patients often retain effective single letter recognition abilities, and develop an effortful letter-by-letter reading strategy which is the basis of most rehabilitation techniques. We study a patient who developed letter-by-letter reading following the surgical removal of left occipito-temporal regions. Using anatomical and functional MRI in the patient and in normal controls, we show that alexia resulted from the deafferentation of left fusiform cortex, and we analyze the network of brain regions subtending letter-by-letter reading. We propose that during letter-by-letter reading (1) letters are identified in the intact right-hemispheric visual system, with a central role for the region symetrical to the VWFA; (2) letters are serially transferred to the left hemisphere through the intact segment of the corpus callosum; (3) word identity is eventually recovered in the left hemisphere through verbal working memory processes involving inferior frontal and supramarginal cortex.
\end{abstract}

(C) 2004 Elsevier Ltd. All rights reserved.

Keywords: Reading; Alexia; fMRI; Fusiform; Language

\section{Introduction}

Pure alexia is a frequent and incapacitating consequence of left occipito-temporal lesions (Binder \& Mohr, 1992; Damasio \& Damasio, 1983). Affected patients suddenly lose expert reading abilities that they have acquired through years of academic training. At the same time, speech comprehension and production, as well as word spelling, are preserved. The essence of this lost perceptual skill is (1) letter identification invariant for position, size, font, and case; and (2) the fast and parallel identification of arrays of several letters (Dehaene et al., 2001; McCandliss, Cohen, \& Dehaene, 2003). It is not before the age of 10 that children show the adult reading pattern (Aghababian \& Nazir, 2000), i.e. short word reading latencies that are independent of word length, at least within a range of about three to seven letters and in optimal display conditions (Lavidor, Ellis, Shillcock, \& Bland, 2001; Weekes, 1997). In severe cases, known as global alexia, patients cannot access abstract letter identity

\footnotetext{
* Corresponding author. Tel.: +33 1 42161849/42161802; fax: +1 44245247 .

E-mail address: laurent.cohen@psl.ap-hop-paris.fr (L. Cohen).
}

(Miozzo \& Caramazza, 1998), and are unable to name even single letters (Dejerine, 1892). More often, alexic patients keep effective letter recognition abilities and develop an effortful letter-by-letter reading strategy, which is the basis of most rehabilitation techniques (Greenwald \& Gonzalez Rothi, 1998). Eventually, this procedure may become quite effective, although it remains easily detectable in the excessive effect of word length on reading latencies (Behrmann, Black, \& Bub, 1990).

A variety of questions and hypotheses have been put forward concerning letter-by-letter reading (for selected references see Montant \& Behrmann, 2000). Does it reflect the partial preservation of normal premorbid processes (Behrmann, Plaut, \& Nelson, 1998), or is it based on novel strategies (Speedie, Rothi, Heilman, 1982)? Is it based on residual left-hemispheric ( $\mathrm{LH})$ or on compensatory right-hemispheric (RH) mechanisms (Coslett \& Saffran, 1998)? Does reveal a general impairment in processing simultaneous visual objects (Farah \& Wallace, 1991) or complex displays (Montant \& Behrmann, 2000), or is it specific to reading? Such issues may be clarified by refering to a simple model of the visual stages of reading (McCandliss et al., 2003). Letters displayed in one hemifield are first an- 
alyzed through a cascade of contralateral retinotopic areas, which compute increasingly abstract representations. Eventually, a representation of letter identities is created in the visual word form area (VWFA), reproducibly located within the left occipito-temporal sulcus, at about TC $-42,-63,-15$ (Cohen et al., 2003). This representation is invariant for parameters such as size, position, case, font. The transfer of visual information from lower-order retinotopic cortices to the VWFA takes place within the left hemisphere for stimuli displayed in the right visual field (RVF). For left visual field (LVF) stimuli, information is conveyed through interhemispheric fiber tracts that course in the splenium of the corpus callosum and over the posterior horns of the lateral ventricles (Binder \& Mohr, 1992; Molko et al., 2002). The VWFA then projects to structures involved in phonological or lexico-semantic processing.

In this framework, pure alexia is thought to result either from the disruption of the VWFA itself, or from impaired projections to or from this system. We recently showed that the critical lesion for pure alexia overlaps accurately with the VWFA, as identified by its activation during normal reading (Cohen et al., 2003). In this study, functional imaging data were obtained in a patient with typical letter-by-letter reading (patient F.). We observed that the intact right-hemispheric cortex symmetrical to the VWFA, which we labeled the R-VWFA, showed a pattern of activation normally specific to the VWFA itself, i.e. stronger activation for alphabetic strings than for chequeboards. Moreover, there were abnormally strong activations in a left frontoparietal network related to verbal working memory. This pattern supported the idea that in letter-by-letter reading, (1) alphabetic symbols are identified in the R-VWFA, (2) they are serially transferred to the left hemisphere, and (3) word identity is recovered by the left hemisphere through an effortful verbal working memory process. Note that this construal is in line with a classical account of letter-by-letter reading (e.g. Binder \& Mohr, 1992; Speedie et al., 1982).

Patient F.'s lesion spared the dorsal bank of the calcarine sulcus, and accordingly the lower right visual quadrant was intact. As we found some residual activations at coordinates close to those of the VWFA, it could not be excluded that a partially spared left-hemispheric pathway leading from V1 to the VWFA could contribute, or even be essential, to letter-by-letter reading. Here we study a young patient (C.Z.) who presented letter-by-letter reading following the surgical resection of left occipito-temporal structures, sparing the VWFA but including the entire primary visual cortex and all fiber tracts leading to the VWFA from both the right and the left visual fields.

Our aims were to assess the account of letter-by-letter reading summarized before, and to fully document a case of pure alexia due to deafferentation of an intact VWFA. Finally, the complete surgical removal of left posterior regions, as opposed to patchy lesions following infarcts, may help to delimit the role of residual left hemispheric structures in letter-by-letter reading.

\section{Medical history and lesion description}

Patient C.Z. was a 19-year-old student, $100 \%$ right-handed according to the Edinburgh inventory (Oldfield, 1971). She was operated twice for the complete resection of a left occipital neuroectodermal tumor, revealed by intracranial hypertension and right hemianopia. Surgery was followed by radiotherapy and chemotherapy. The present study was carried out between 6 and 12 months after the surgery. There was no indication of relapse on close clinical and radiological follow-up. Following the intervention, the patient complained of being unable to read, while she could write normally. Her family also reported substantial memory difficulties.

The anatomical MRI showed that the left occipital lobe had been removed, including the entire striate cortex and the left half of the splenium of the corpus callosum (Fig. 1). The most anterior part of the lateral occipital cortex was spared. The excision extended to the ventral and mesial temporal lobe, as well as to the underlying white matter, reaching TC $y=-40$ anteriorily. In the temporal lobe, the lesion affected the hippocampal and parahippocampal gyri, and stopped at the mesial edge of the fusiform gyrus.

\section{Behavioural assessment}

\subsection{General data}

Goldmann perimetry showed complete right hemianopia without sparing of the macula. There was a marked memory impairment, in the verbal more than in the visual domain: with the Wechsler Memory Scale Revised, she scored 9/50 $(-2.4 \sigma)$ and $0 / 50$ on the immediate and delayed verbal memory tests; and $36 / 41(+0.5 \sigma)$ and $23 / 41(-1 \sigma)$ on the immediate and delayed visual memory tests. Tests for color naming and object-color matching, as well as Benton's face matching test were performed flawlessly. Oral language production, comprehension, and repetition were normal. There were minimal word-finding difficulties $(91 / 100$ correct on the DENO 100 picture-naming test). C.Z. was asked to write to dictation a list of 36 frequent words (three to nine letters and one to four syllables in length), and to identify another set of 36 similar words orally spelled-out by the examiner. She was flawless in the dictation test. She made 2/36 errors in the word identification test, proposing responses close to the target (e.g. MUSIQUE $\rightarrow$ Munich).

\subsection{Reading}

The patient was asked to read aloud a short story of about 15 lines. She made essentially no errors (pâte $\rightarrow$ pâté), but it took her more than 11 min to complete this task, while normal subjects need on average $35 \mathrm{~s}$ (Cohen et al., 2003).

Six months after surgery, C.Z. was presented with 79 lower-case familiar nouns, three to nine letters and one to 


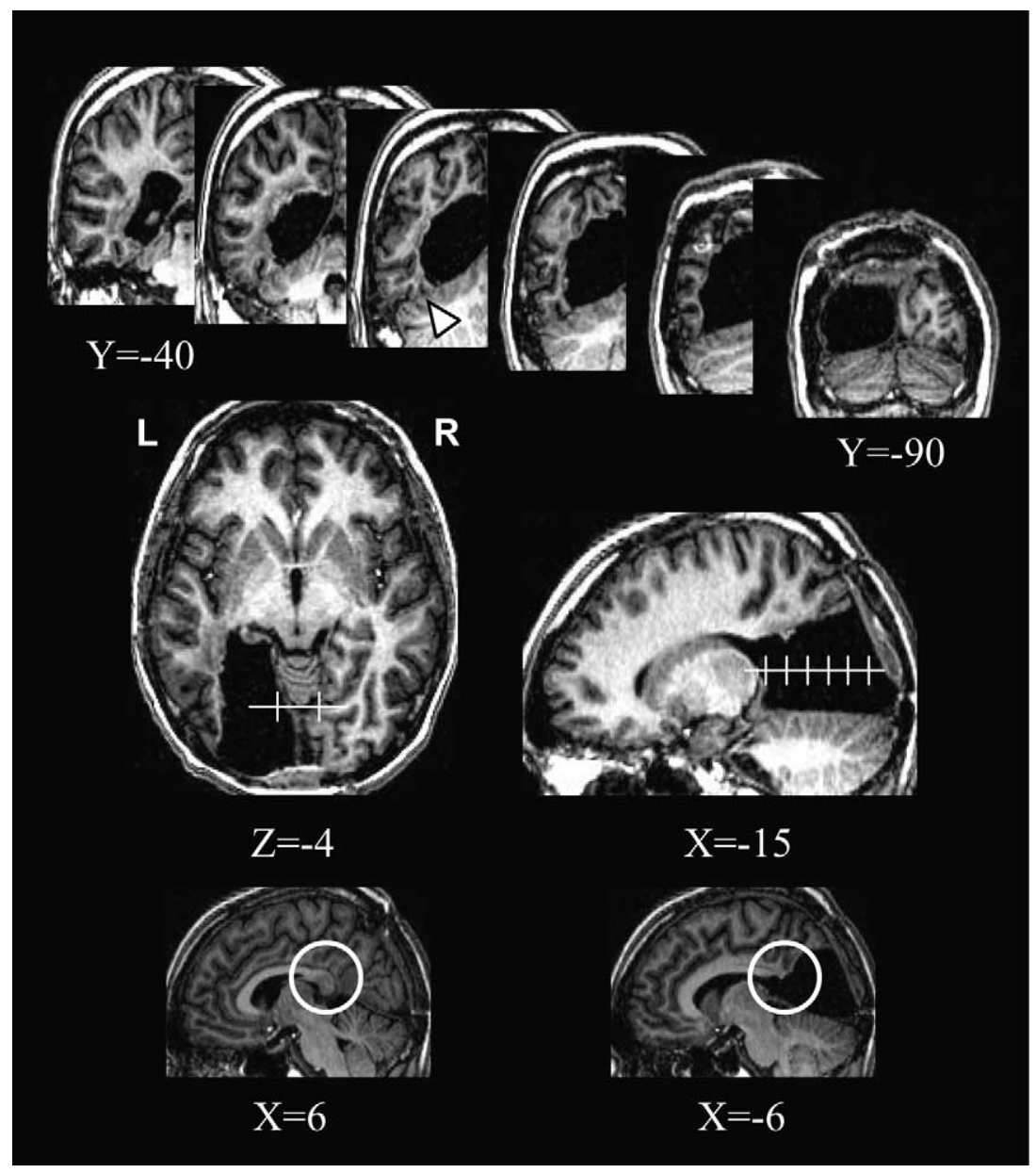

Fig. 1. Normalized T1-weighted MRI of patient C.Z., showing the surgical resection of most of the left occipital lobe including the left half of the splenium, the posterior hemispheric white matter, the hippocampal and parahippocampal gyri. The white arrowhead points to the spared mid-fusiform cortex.

four syllables in length. Stimuli were presented centrally on a computer screen, subtending a maximum angle of $4^{\circ}$ on each side of fixation, using the Courier New font. Stimuli remained visible until a response was produced. Latencies were measured using a voice key. C.Z. was asked to read each word aloud as rapidly as she could, while minimizing errors. She made no errors, but her mean reading time was $8196 \mathrm{~ms}$ (excluding 2 outliers beyond $20 \mathrm{~s}$ ), and was strongly correlated with word length (Fig. 2), with a slope of $1181 \mathrm{~ms}$ per letter $(r(5$ d.f. $)=0.94 ; P<0.002)$. Five months later, at about the time of the imaging experiment, her speed had improved, as reading the short text took her 7 min, while the slope of the word length effect had diminished to $431 \mathrm{~ms}$ per letter.

All upper- and lower-case letters were presented randomly using the same technique. C.Z. made no error out of 52 trials, but was significantly slowed down (mean reading time $=$ $1964 \mathrm{~ms}$, excluding one outlier at $12 \mathrm{~s}$ ). In order to assess her mental imagery for letters, the patient was orally presented with the name of all letters, and was asked to decide whether the upper-case letter included a curve or not. She made no error. In a similar task, she was asked to decide

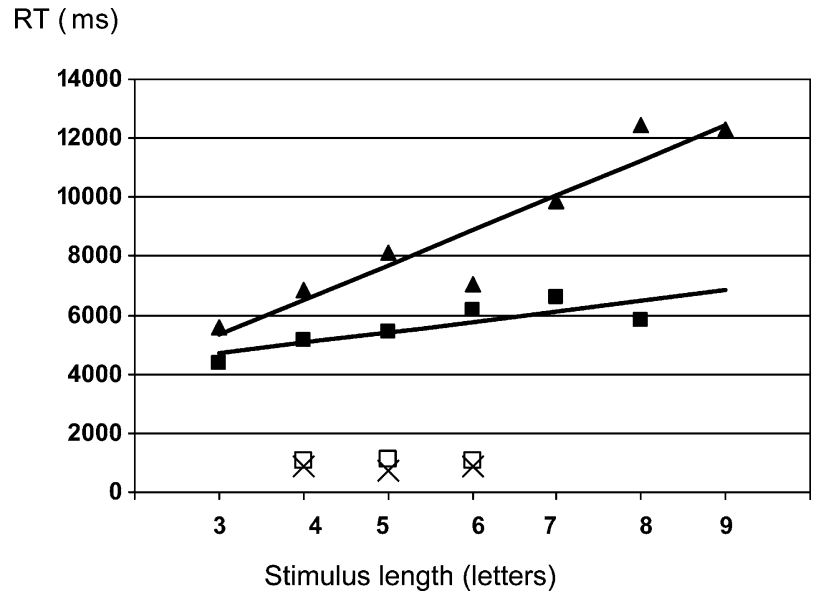

Fig. 2. Patient C.Z.'s correct response latencies in the initial word reading test (triangles), in word reading at the time of the imaging experiment (solid squares), and in lexical decision (real words: crosses; consonant strings: open squares). She showed a letter-by-letter reading pattern, with a slope of $1181 \mathrm{~ms}$ per letter. In contrast, she could discriminate real words from consonant strings rapidly and with no effect of length. 
whether lower-case letters included a descender ( $j, g, p, q$, y). She made $4 / 26$ errors, all corresponding to an initial misunderstanding of instructions.

In order to assess the access to abstract letter identity, two further tests were proposed to C.Z., using only letters with widely different upper-case and lower-case shapes. First, she was presented with 10 upper-case and 10 lower-case letters and was asked to write down each letter in the opposite case. She made a single error $(q \rightarrow G)$. Second, the patient was presented with 80 pairs of letters horizontally displayed on a computer screen. The two letters in a pair were printed in different cases, and C.Z. had to decide whether the two letters corresponded to the same grapheme (e.g. a A) or not (e.g. a B), and to respond using one of two keys. C.Z. made $2 / 80$ errors. In order to make it more sensitive to subtle impairments of letter processing, this task was run again, with stimuli displayed for only $200 \mathrm{~ms}$ and followed by a masking pattern (\#\#\#\#\#). The patient made 24/80 errors. However, as this high error rate could be due to hemianopia, the test was run once again, with the two letters displayed one above the other. C.Z. still made 12/80 errors, with a bias for responding "different".

Finally, the patient was submitted to lexical decision tasks. She was first asked to discriminate words versus strings of consonants. She was presented with three lists of 30 items each. Each list comprised 15 frequent words $\left(\log _{10}\right.$ frequency per million >2), 4-6 letters long, and 15 consonant strings matched in length to the words. Stimuli were displayed for $200 \mathrm{~ms}$ and followed by a masking pattern (\#\#\#\#\#). C.Z. had to decide whether stimuli were real words or not, and to respond using one of two keys. The patient judged the task to be easy, explaining that she could instantly pick out large consonant clusters, and she performed way above chance (17/90 errors; $19 \% ; P<0.0001)$. She had a mean RT of $947 \mathrm{~ms}$, and latencies did not increase with word length, suggesting that, in this task, the patient did not resort to letter-by-letter reading (Fig. 2). To further clarify whether the patient could indeed detect clusters of consonants beyond the reach of letter-by-letter reading, two lists of six-letter stimuli were created, for which the decision could be based only on the last three letters. The first list, which was run three times, included 12 words with three initial consonants, and 12 matched consonant strings with the same first three letters (e.g. SPHERE versus SPHCRQ). The second list, which was run twice, included 25 frequent words, and 25 matched non-words, with the same first three letters but three final consonants (e.g. BONDIR versus BONDFL). Rare letters such as $\mathrm{K}, \mathrm{X}, \mathrm{Z}$ were excluded. The patient responded better than chance with both lists (21/72 errors; $29 \% ; P<0.025$; and 35/100 errors; 35\%; $P<0.05$, respectively), confirming that she could pick out consonant clusters better than expected on the basis of left-to-right letter-by-letter reading.

In summary, the patient showed typical pure alexia, with a sparing of oral language and of spelling abilities. She resorted to letter-by-letter word reading, with a slope of read- ing latencies that was initially over $1 \mathrm{~s}$ per letter. The identification of single letters was accurate, but abnormally slow, with errors in a cross-case matching task under short display conditions. Finally, C.Z. could apparently spot consonant strings with relatively good accuracy and speed. She was indeed faster at detecting consonant strings than at reading the shortest words, or even single letters.

\subsection{Brain imaging}

The patient was submitted to the same imaging procedure and compared to the same group of nine normal controls as described in Cohen et al. (2003).

\subsection{Procedure}

The patient was presented with four types of stimuli: a fixation point, real words, consonant strings, and chequerboards covering approximately the same field as letter strings. The 128 words were a subset of the list used for the behavioural assessment, including 38 three to four words, 60 five to seven letter words, 30 eight to nine letter words. Consonant strings were matched one-to-one to real words in number of letters. The frequency distribution of consonants was the same in consonant strings as in words. Stimuli were presented centrally for $1700 \mathrm{~ms}$, followed by a $800 \mathrm{~ms}$ fixation point. The patient was instructed to pay attention equally to all types stimuli and to read real words covertly.

The patient received four fMRI sequences, each comprising 16 experimental blocks, i.e. four blocks of each condition. Each block comprised eight trials. Blocks were presented in pseudo-random order within sequences, so as to maximize the variety of transitions between conditions while avoiding any repetition of the same condition in successive blocks. Trials were presented in random order within blocks. The order of blocks within sequences and the order of trials within blocks differed across the four sequences.

\subsection{Imaging parameters}

Each sequence consisted of $10 \mathrm{~s}$ of initial fixation, followed by 16 stimulation blocks of $20 \mathrm{~s}$ each, as described before. In each sequence, 132 functional volumes sensitive to blood oxygen level dependent (BOLD) contrast were acquired with a T2-weighted gradient echo, echo planar imaging sequence on a 1.5 tesla Signa Imager (TR (relaxation time $)=2500 \mathrm{~ms}, \alpha$ (flip angle) $=90$, TE (echo time) $=60 \mathrm{~ms}$, field of view $=240 \mathrm{~mm} \times 240 \mathrm{~mm}$, in plane resolution $=3.75 \mathrm{~mm} \times 3.75 \mathrm{~mm}$ ). Each volume comprised 17 axial slices of $5 \mathrm{~mm}$ thickness covering most of the brain. The first four volumes were discarded to reach signal equilibrium. High-resolution T1-weighted images (3D fast gradient-echo inversion recovery sequence, TI (inversion time) $=400 \mathrm{~ms}, \mathrm{TR}=11 \mathrm{~ms}, \mathrm{TE}=2 \mathrm{~ms}, \alpha=20$, field of view $=240 \mathrm{~mm} \times 240 \mathrm{~mm}$, slice thickness $=1.5 \mathrm{~mm}$, 
in-plane resolution $=0.94 \mathrm{~mm} \times 0.94 \mathrm{~mm}$ ) were also acquired for anatomical localization, as well as T2-weighted fast spin echo and FLAIR axial slices.

\subsection{Analysis of MRI data}

Functional images were analysed with the Statistical Parametric Mapping software (SPM99). To correct for motion, functional scans were realigned using the first image as a reference. As functional images were locally distorted by metallic surgical material, functional images were transformed to TC (Talairach \& Tournoux, 1988) using the standard EPI template, applying nonlinear normalization parameters while masking the lesion and the magnetic artifact (Brett, Leff, Rorden, \& Ashburner, 2001). Functional images were then smoothed with a Gaussian spatial filter ( $5 \mathrm{~mm}$ FWHM). The resulting images had cubic voxels of $3 \mathrm{~mm} \times 3 \mathrm{~mm} \times 3 \mathrm{~mm}$. The anatomical image was linearly transformed to TC using the standard T1 template.

Activation on each of the 4 types of trials was modeled by a combination of the standard SPM haemodynamic function and its temporal derivative. Only the former function was used for statistical contrasts. Four additional variables of non-interest modelled constant differences across the four sequences. Long-term signal variations were eliminated with a high-pass filter set at $240 \mathrm{~s}$. Low-pass filtering was achieved by convolution with the haemodynamic response function. In individual analyses, we used an uncorrected voxelwise threshold of $P<0.001$, a clusterwise threshold of $P<0.05$ corrected, and all peak voxels mentioned in the descriptions of activation networks had a statistical significance of $P<10^{-5}$, unless stated otherwise.

\section{Results}

We will first delineate the overall network of activated areas, then concentrate on regions involved in processing alphabetic stimuli, and eventually focus on letter-by-letter reading by contrasting words versus consonant strings. We put an emphasis on occipito-temporal regions, and particularly on the distribution of activations across the lesioned left-hemispheric and the intact right-hemispheric cortices. Whenever relevant, individual analyses of the patient's data are supplemented with comparisons to the group of normal controls.

\subsection{Overall reading network}

\subsubsection{Alphabetic stimuli versus fixation}

In order to identify the overall reading network, we contrasted alphabetic stimuli (i.e. words and consonant strings) versus fixation (Fig. 3). Ventral occipito-temporal activations will be reported last.

There were bilateral activations in the horizontal (left: TC $-30,-57,51 ; Z>15$; right: TC $27,-48,54 ; Z>15$ ) and posterior (left: TC $-21,-66,30 ; Z>15$; right: TC 33 , $-63,21 ; Z>15)$ segments of the intraparietal sulcus; in the SMA/cingulate (TC $-3,9,57 ; Z>15$ ); in the dorsolateral (left: TC $-27,42,15 ; Z=6.68$; right: TC 21, 69, 24; $Z=6.87$ and TC $304530 ; Z=7.55$ ) and orbital (left: TC $-3345-24 ; Z>10$; right: TC $12,63,-15 ; Z=7.30$ ) prefrontal cortex; in the precentral cortex (left: TC $-48,0$, $30 ; Z>15$; right: $51,21,33 ; Z>10$ ); in Broca's area and its right-sided counterpart (left: TC $-42,27,0 ; Z>9$; right: TC $48,24,-9 ; Z=6.59)$. Although bilateral, most of those activations showed left-hemispheric predominance. Additionally there were left-sided activations in the supramarginal gyrus (SMG) (TC $-57,-33,33 ; Z=7.84)$ and the thalamus (TC $-12,-18,6 ; Z>8)$.

In the $\mathrm{LH}$, there was a residual stripe of activation in the ventral occipito-temporal cortex just lateral to the lesion. This activation followed the fusiform gyrus in its posterior (TC $-36,-84,-12 ; Z=4.60 ;$ TC $-33,-75,-18 ; Z>$ 10 ) and middle segment (TC $-33-57-15 ; Z=6.07$ ), and extended anteriorily to TC $Y=-39$. Note that the peak at TC $-33,-57,-15$ was close to the normal coordinates of the VWFA.

The symmetrical activations in the RH were more extensive, and the intensity of activations as measured by the percent change of BOLD signal was about three times higher than in the left-hemispheric ventral temporal cortex (Fig. 3 ). Within the right parieto-occipito-temporal cluster, ventral activations started from the occipital pole (TC 24, -96, -18; $Z>20$ ), and followed the fusiform gyrus in its posterior (TC $42,-87,-21 ; Z>15$ ) and middle segment (TC 51, -69 , $-21 ; Z>9$ ), again extending to TC $Y=-39$. This stripe included the region symmetrical to the VWFA. Slightly more dorsally there were activation foci in the mesial (TC 21-81, $0 ; Z>9$ ) and lateral (TC 39-81, $-6 ; Z>15$ ) posterior occipital cortex.

\subsubsection{Alphabetic stimuli versus chequerboards}

In order to identify within this network which regions were more specifically involved in processing alphabetic stimuli, we compared alphabetic stimuli versus chequerboards, while masking by words vs. chequerboards and consonants vs. chequerboards $(P<0.01$ each). This analysis revealed a large network almost encompassing the entire network identified relative to fixation.

In the left occipito-temporal region, there was again a stripe of activation following the fusiform gyrus from its posterior segment (TC $-36,-84,-21 ; Z=5.63$; $\mathrm{TC}-33$, $-75,-18 ; Z=7.73)$ to TC $Y=-57$. In the RH, activations did not start from the occipital pole, but from the posterior segment of the fusiform gyrus (TC $45-87-21 ; Z>15$ ), extended to its middle portion (TC $54,-69,-21 ; Z=6.45$ ) including the region symmetrical to the VWFA (TC 39, -51 , $-27 ; Z=6.65$ ), and anteriorily to TC $Y=-45$. Note that the occipital pole did not appear in this contrast because it was equally activated by chequerboards (TC $24,-93,-18$; $Z>15$ ) and alphabetic stimuli relative to fixation (Fig. 3). 


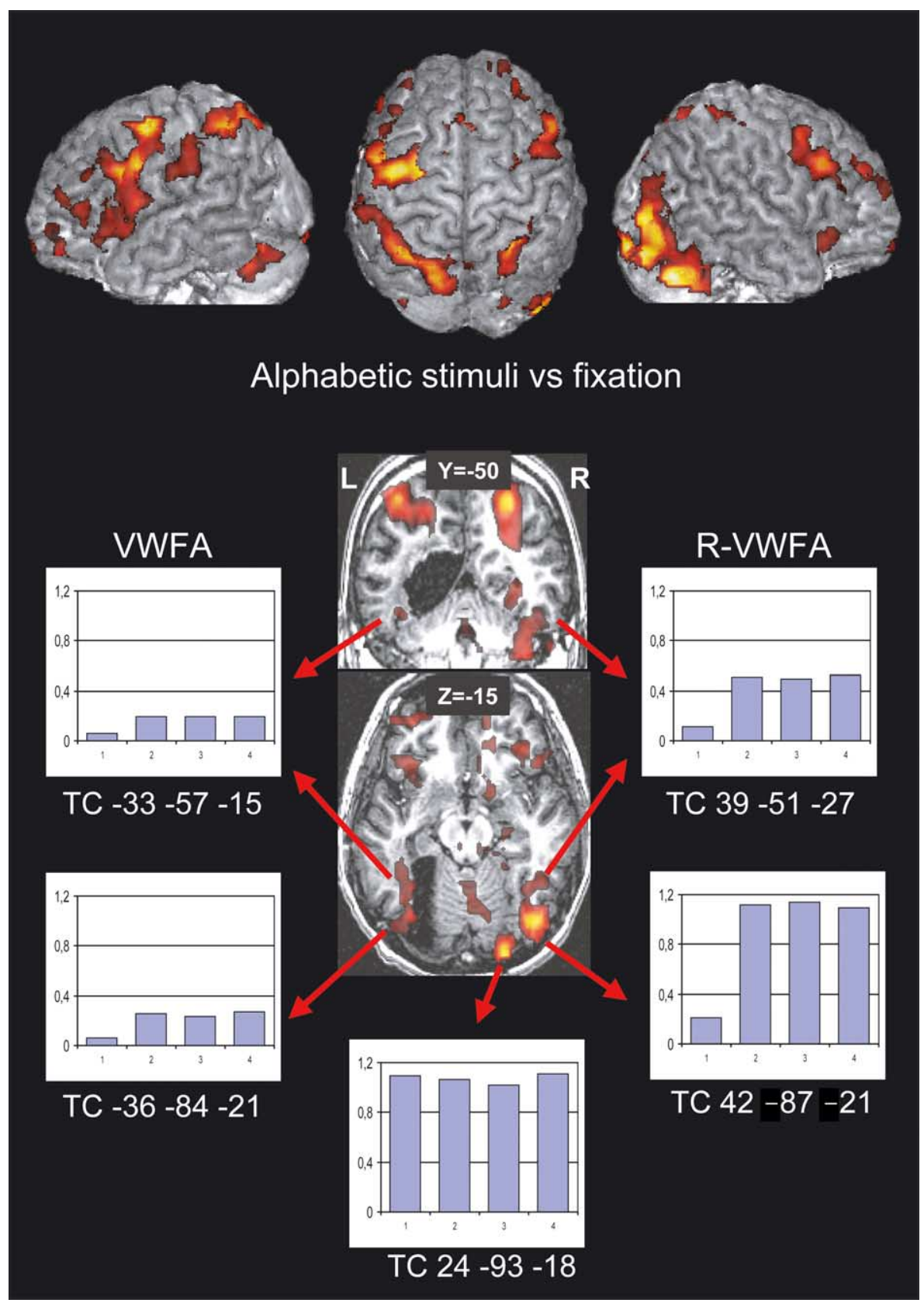

Fig. 3. Top: General reading network as revealed by the contrast of alphabetic stimuli versus fixation, overlaid on a 3D render of the patient's brain. Bottom: Percent change in BOLD signal relative to fixation in selected occipito-temporal voxels. Histogram bars correspond to chequerboards, alphabetic stimuli, consonant strings, and real words, respectively. Except for the right posterior occipital cluster, all regions showed a stronger activation for alphabetic stimuli than for chequerboards. Contrary to the normal pattern, activations were about 3 times stronger in the right than in the left hemisphere, and the R-VWFA showed stronger activations for alphabetic stimuli than for chequerboards.

\subsubsection{Occipito-temporal activations in the patient and in controls}

Thus two anomalies were observed in the patient's occipito-temporal activations. First, in normals, contrasting alphabetic stimuli versus fixation elicits roughly symmetrical ventral occipito-temporal activations. In the patient, activations were three times stronger in the right than in the left hemisphere, in the cortex just lateral to the lesion. Second, in normals, contrasting alphabetic stimuli versus che- querboards showed exclusively left-sided occipito-temporal activations. In the patient however, such activations predominated in the right hemisphere.

In order to assess the abnormality of this pattern, we compared the contrast of alphabetic stimuli versus chequerboards in the patient relative to the group of controls (voxelwise $P<0.02 ; P<0.05$ for cluster extent corrected within the patient's overall reading network). This showed right-hemispheric activation in the occipital cor- 
tex (TC 33, $-63,15 ; Z=5.18$ ), the posterior fusiform gyrus (TC $45,-78,-12 ; Z=4.83$ ), and extending in the parahippocampal region (TC $27,-48,-6 ; Z=3.08$ ). There was also a small cluster in the region symmetrical to the VWFA (TC 42, -54, $-27 ; Z=2.73$ ) below the extent threshold. There was no activation in the corresponding left-hemispheric regions. This confirms that the patient's right occipito-temporal cortex symmetrical to the VWFA was strongly and abnormally involved in the processing of alphabetic stimuli, as opposed to simple geometrical patterns.

\subsection{Letter-by-letter reading: words versus consonant strings}

Beyond an increased reliance on right temporal cortex for alphabetic processing, letter-by-letter reading should require a more extensive network of areas in order to progressively figure out word identity. A critical contrast to delimit this network is the difference between real words and consonant string (Fig. 4). Indeed, the patient could spot rapidly and accurately consonant strings, and she therefore engaged fully in letter-by-letter reading with real words
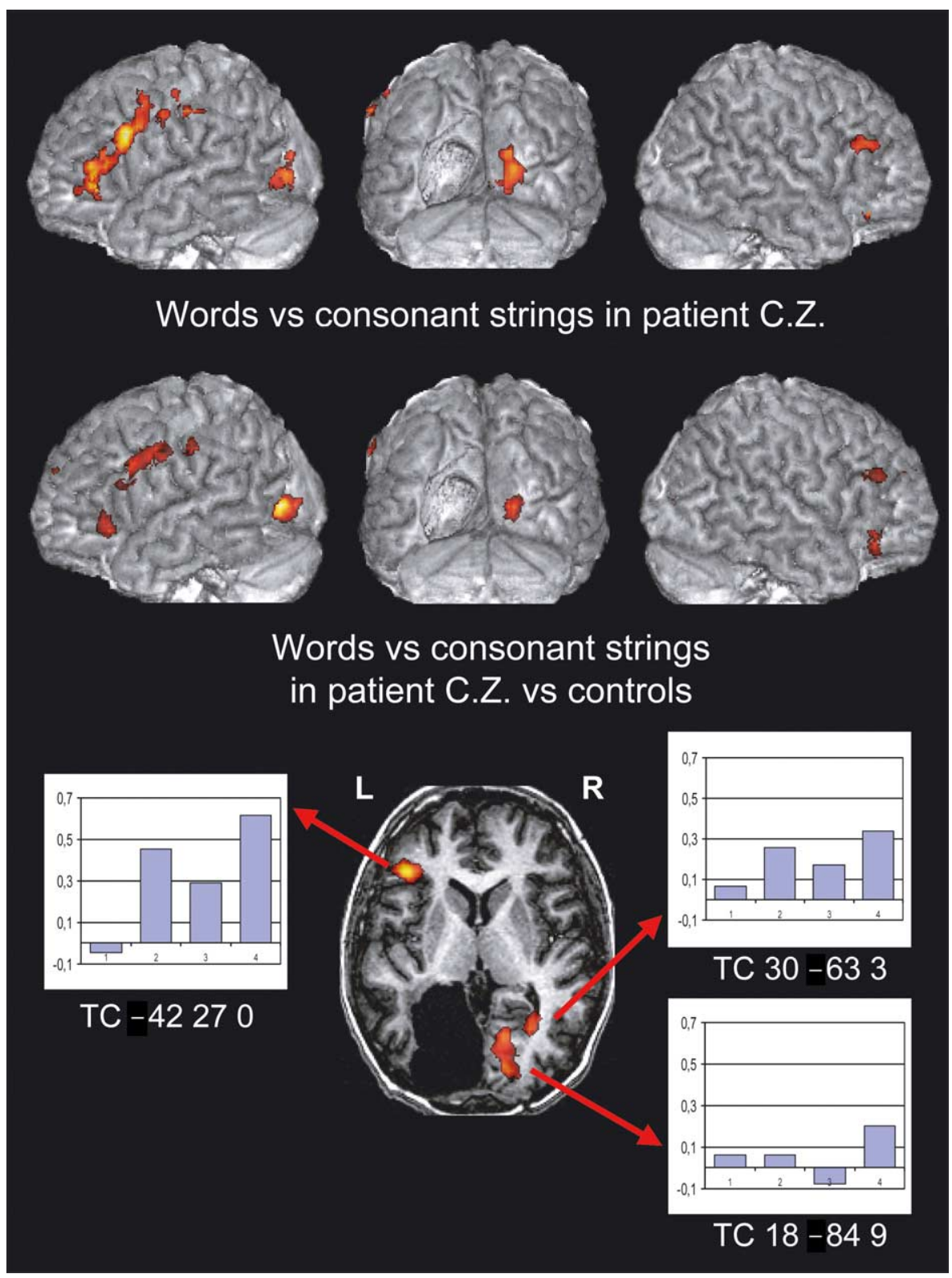

Fig. 4. Top: Right occipital, predominantly left frontal, and left parietal regions activated by words vs. consonant strings, and associated with letter-by-letter reading. Middle: The same regions are found when comparing activations by words vs consonant in the patient relative to normal controls. Note that right occipital activations are visible on the left lateral view due to the resection of the left occipital pole. Bottom: Percent change in BOLD signal relative to fixation in selected voxels with stronger activation for words than for consonants. 
only. Note that according to her own report, the patient was not always sure of having correctly identified target words, a limitation which could be expected considering the relatively rapid rate of stimulation. However, she always attempted to identify words through letter-by-letter reading. The activation patterns reported now confirmed that words and consonant strings were actually processed differently.

The subtraction of words minus consonant strings showed left hemispheric activations in the left precentral cortex (TC $-60,9,33 ; Z=6.53$ ), Broca's area (TC $-42,27,0 ; Z=$ 6.04 ), and the SMG (TC $-66,-27,42 ; Z=5.27$; clusterwise $P<0.1$ corrected). There were bilateral activations in the SMA (left: TC $-9,21,54 ; Z=4.81$; right: TC 3, 30, 51; $Z=3.98$; clusterwise $P<0.06$ corrected). In the RH, there were activations in the dorsolateral (TC 45, 24, 21; $Z=4.33$ ) and mesial (TC 9, 45, 21; $Z=4.46$; clusterwise $P<0.06$ corrected) prefrontal cortex; in the inferior frontal gyrus (TC 30, 27, $-12 ; Z=5.74$ ); and in the mesial occipital cortex (TC 18, $-84,9 ; Z=5.06$; and TC 30, $-63,3$; $Z=4.42)$.

In order to assess statistically the deviation of this pattern from the norm, we compared the contrast of words versus consonants in the patient relative to the group of controls (voxelwise $P<0.02 ; P<0.05$ for cluster extent corrected within the patient's overall reading network). This procedure showed a network essentially identical to the previous analysis (Fig. 4): left precentral (TC $-57,9,30 ; Z=3.51$; and TC $-39,-3,30 ; Z=3.34$ ), Broca's area (TC $-33,21,-9$; $Z=3.80$ ), right inferior frontal (TC 33, 30, $-9 ; Z=3.75$ ), and right occipital activations (TC 18, $-78,6 ; Z=4.40$; and TC 30, -60, 0; $Z=3.85$ ) (all Ps $<0.001$ ). Activations in the left SMG (TC $-66,-24,39 ; Z=3.51 ; P<0.001$ ), bilateral mesial frontal cortex, and right dorsolateral prefrontal were also present but too small to reach clusterwise significance.

\section{Discussion}

\subsection{Pure alexia due to a disconnected VWFA?}

We proposed that the VWFA, as identified in a number of functional studies using PET or fMRI (Beauregard et al., 1997; Brunswick, McCrory, Price, Frith, \& Frith, 1999; Fiez, Balota, Raichle, \& Petersen, 1999; Cohen et al., 2000, 2002; Dehaene et al., 2001; Dehaene, Le Clec'H, Poline, Le Bihan, \& Cohen, 2002; Paulesu et al., 2000; Wagner et al., 1998), MEG or intracerebral recordings (Allison, McCarthy, Nobre, Puce, \& Belger, 1994; Allison, Puce, Spencer, \& McCarthy, 1999; Cohen et al., 2000; Nobre, Allison, \& McCarthy, 1994; Salmelin, Service, Kiesilä, Uutela, \& Salonen, 1996; Tarkiainen, Helenius, Hansen, Cornelissen, \& Salmelin, 1999; Simos et al., 2002) plays a key role in reaching an abstract visual representation of letter strings, independent from position, font, or case, on the basis of fast and parallel letter identification. In this framework, a selective disruption of the VWFA should result in an isolated reading deficit, in the absence of other language impairments, i.e. pure alexia. The current evidence from anatomo-clinical correlations is compatible with this hypothesis, as it points to the mid-portion of the left fusiform gyrus as the critical lesion site for pure alexia (Beversdorf, Ratcliffe, Rhodes, \& Reeves, 1997; Binder \& Mohr, 1992; Cohen et al., 2003; Damasio \& Damasio, 1983).

However, a close analysis of patient C.Z.'s lesion suggests that it actually spared the fusiform cortex and the VWFA (Fig. 1). In Talairach normalized space, the peak activation of the VWFA in control subjects falls at about TC -42 , $-63,-15$ (Cohen et al., 2003). At this anteroposterior level $(y=-63)$, patient C.Z.'s lesion extended laterally only to $x=-28$, that is more mesial than the VWFA of any normal subject (Fig. 5). This point is confirmed by considering anatomical landmarks rather than normalized coordinates. In individual analyses, the VWFA consistently falls in the depth of the occipito-temporal sulcus, lateral to the fusiform gyrus (Cohen et al., 2000; Cohen et al., 2002; Dehaene et al., 2002; Gauthier et al., 2000; Puce, Allison, Asgari, Gore, \& McCarthy, 1996), a region that was clearly spared in patient C.Z. Finally, the patient showed residual activations at the normal coordinates of the VWFA.

Considering that the VWFA itself was spared, we propose that the reading deficit resulted from its deafferentation from visual input. As visible on Fig. 1, the left occipital lobe and the occipito-temporal white matter were extensively removed, including callosal connexions, depriving the remaining fusiform gyrus of input connections from both left- and right-hemispheric lower-level visual cortex (Catani, Jones, Donato, \& Ffytche, 2003). Indeed, the patient's white matter lesion encompassed and far exceeded the typical callosal lesion responsible for alexia in the left visual hemifield (Suzuki et al., 1998). In such cases, deafferentation of the VWFA from right-hemispheric input was demonstrated by a reduced activation by left-sided as compared to right-sided words (Cohen et al., 2000; Cohen et al., 2003). Moreover, the interhemispheric pathway critically involved in hemialexia, as delineated using diffusion tensor imaging, falls within the region that was resected in the present case (Molko et al., 2002). As a point of history, one may note that the original alexic patient reported by Dejerine (1892) also had an intact mid-fusiform cortex, while the lesion affected the underlying white matter (Dejerine, 1895).

\subsection{Role of residual left fusiform activations}

Although patient C.Z.'s VWFA was deprived of visual input, it was significantly activated during the imaging experiment. However, those activations were much weaker than their right-hemispheric counterpart, due to cortical excision and to the disconnection of remaining cortex. Their functional meaning remains unclear. It should be stressed that C.Z.'s pattern of alexia was perfectly indistin- 


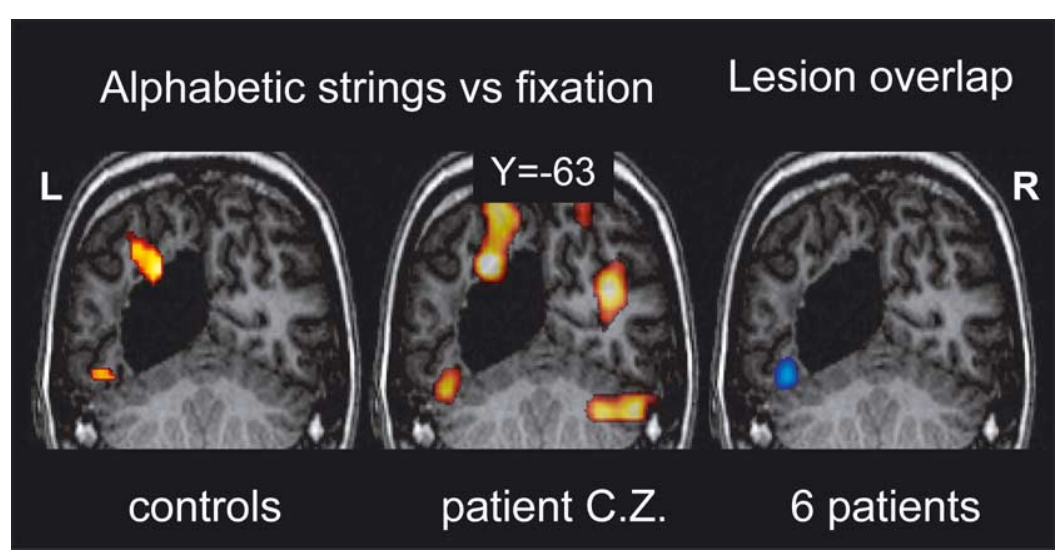

Fig. 5. Patient C.Z.'s lesion was just mesial to the left fusiform gyrus, thus sparing the VWFA, as identified by its activation in normal controls (left), by its residual activation in patient C.Z. (middle), and by the overlap of cortical lesions in patients with pure alexia (right; Cohen et al., 2003).

guishable from other more common patients with cortical damage actually destroying the fusiform cortex, like for instance patients reported by Cohen et al. (2003). This demonstrates that the preservation of an intact but deafferented fusiform cortex makes no difference as regards reading performance. It thus seems safe to conclude that the patient's VWFA played no causal role in letter-by-letter reading.

The residual activation of the VWFA probably resulted from top-down influences reaching the VWFA through preserved long-distance and U-shaped association fibers. Indeed ventral areas devoted to object recognition can be activated in the absence of visual input during mental imagery (O'Craven \& Kanwisher, 2000). Patient C.Z. was excellent in tasks of mental imagery involving letter shapes, an ability to which the VWFA possibly contributed (for an opposite case see Bartolomeo, Bachoud-Levi, Chokron, \& Degos, 2002). There is also evidence that infero-temporal regions can receive top-down activation by auditory words, whenever orthographic processing is required. Thus Burton, Small, \& Blumstein (2000) observed a left infero-temporal activation (TC $-58,-56,-8)$ when subjects performed same-different judgments on spoken syllables, only when they had to extract the first phoneme of the syllable, but not when their decision was based on the syllable as a whole. The former task is not within the reach of illiterate subjects, and requires reading-dependent phonological awareness (Morais \& Kolinsky, 1994). Similarly, Booth et al. (2002, 2003) observed an activation of the left VWFA (TC -45 , $-57,-12$ ) when subjects engaged in a spelling task on auditory words, but not when they performed a rhyming task on the same stimuli. It is an open question whether the residual activations of the patient's VWFA could be modulated by such task manipulations.

In summary, patient C.Z.'s VWFA was anatomically spared, but deafferented of all visual input, explaining the occurrence of pure alexia. Residual top-down activations were observed in the VWFA, but they probably played no actual role in letter-by-letter reading.

\subsection{The mechanisms of letter-by-letter reading}

One may a priori assume that efficient letter-by-letter reading requires the cooperation of a letter identification device, presumably located in the ventral visual stream, and of a verbal working memory network in charge of combining letters and of accessing word identity, presumably involving left-hemispheric perisylvian fronto-parietal structures. The functional imaging study of patient C.Z. allowed us to flesh out these hypotheses, which will be discussed in turn.

\subsection{Letter identification and the right occipito-temporal cortex}

We argued that although the VWFA itself was spared by the resection, it was deprived of any visual input and probably played no causal role in letter-by-letter reading. The right-sided occipito-temporal cortex, including the RVWFA, appears as a likely substitute for the purpose of identifying visual letters. There is indeed convergent evidence that the right hemisphere is able to identify letters, from studies of split-brain patients (Baynes \& Eliassen, 1998), patients with left hemispherectomy (Patterson, Vargha-Khadem, \& Polkey, 1989), or deep dyslexia from large left-sided lesions (Coltheart, 1980). Furthermore, the residual reading abilities of pure alexic patients may be lost following disruption of right occipito-temporal systems, either following a subsequent right-sided lesion (Bartolomeo, Bachoud-Levi, Degos, \& Boller, 1998), or by means of transcranial magnetic stimulation (Coslett \& Monsul, 1994).

Regarding the specific involvement of the R-VWFA, our hypothesis is supported by the abnormal pattern of activation of this region observed in patient C.Z. First, the RVWFA was activated 3 times more strongly by alphabetic stimuli relative to rest than the VWFA proper, while activations are roughly symmetrical in normal subjects. Second, the R-VWFA and more posterior right fusiform cortex were more activated by alphabetic stimuli than by chequerboards, a key functional feature of the normal VWFA. This pattern 
was similar to what was observed in another letter-by-letter reader (Cohen et al., 2003). That the R-VWFA should take up letter identification probabaly does not imply a thorough modification of its functional properties. Indeed, in the protocol used with patient C.Z., normal controls, the R-VWFA is activated by alphabetic stimuli relative to fixation with almost the same strength as the VWFA. This suggests that, in the right hemisphere also, letters are encoded in highlevel ventral visual cortex devoted to object recognition. A left-hemispheric predominance emerges most clearly when contrasting letter strings versus higher-level visual controls such as chequerboards (Cohen et al., 2002), textures (Puce et al., 1996), faces (Gauthier et al., 2000; Puce et al., 1996), buildings (Hasson, Levy, Behrmann, Hendler, \& Malach, 2002), or pseudoletters (Price, Wise, \& Frackowiak, 1996). Some of the specificities of the VWFA as compared to the R-VWFA may be that the VWFA builds up a representation of letter strings more invariant for changes of case (Dehaene et al., 2004; Dehaene et al., 2001; see also Koutstaal et al., 2001) and of spatial position (Cohen et al., 2000; Cohen et al., 2002; Hasson et al., 2002), and is more selectively tuned to alphabetic stimuli than to other types of objects (Hasson, Harel, Levy, \& Malach, 2003).

Hence one may expect that the substitution of the VWFA by the right-hemispheric structures should not be perfect. This may explain the patient's abnormal performance in demanding letter processing tasks, particularly in the crosscase matching task with fast stimulus presentation. If indeed the VWFA is the key system for computing the equivalence between upper- and lower-case letters (Dehaene et al., 2001; Polk \& Farah, 2002), its disruption is likely to make the task difficult, as in C.Z., or even impossible in other alexic patients (Chanoine, Teixeira Ferreira, Demonet, Nespoulous, \& Poncet, 1998; Miozzo and Caramazza, 1998).

In addition to the right fusiform, the right primary visual cortex emerged as a likely component of letter-by-letter reading in patient C.Z. Contrary to what prevails in normal controls, early visual cortices were activated more strongly by words, which require letter-by-letter reading, than by consonant strings, which the patient could rapidly pick out. A number of imaging studies have shown that attentional control modulates the activation of the primary visual cortex (for a review see Pessoa, Kastner, \& Ungerleider, 2003). Thus attended stimuli elicit stronger activations in striate and extrastriate cortex than non-attended stimuli (Muller, Bartelt, Donner, Villringer, \& Brandt, 2003; Somers, Dale, Seiffert, \& Tootell, 1999). Attentional amplification was also evidenced in patients with visual extinction, in whom consciously perceived stimuli elicited stronger activations than extinguished stimuli in the fusiform gyrus but also in the primary visual cortex (TC 8, -94, 0) (Vuilleumier et al., 2001). It may be relevant to reading that when multiple object are presented simultaneously, they exert mutual inhibition, resulting in decreased visual activations relative to sequential presentation (Kastner, De Weerd, Desimone, \& Ungerleider, 1998). However, directing attention towards one of the stim- uli compensates this reduction of activity. Similarly, one may speculate that the mutual inhibition among simultaneously displayed letters is compensated by successively attending to the letters in the stimulus word, explaining increased activations during letter-by-letter reading. Finally, the increased occipital activation may also reflect the contribution of early visual cortex to the storage of visual information in working memory during letter-by-letter reading (Super, Spekreijse, $\&$ Lamme, 2001).

\subsection{Verbal working memory and the left perisylvian cortex}

A central component of letter-by-letter reading is the piecemeal assembly and identification of the target word, based on the serial recognition of letters. This may require a variety of operations, such as the storage in short term memory of an increasing set of letters, their phonological recoding, their combination into larger units, repeated access to the lexicon, etc. Accordingly, patient C.Z. showed stronger than normal activations in areas related to language and verbal working memory: Broca's area, the left SMG, mesial and dorsolateral prefrontal cortex (for reviews of activations see Cabeza \& Nyberg, 2000; D'Esposito, Postle, \& Rypma, 2000), in agreement with Cohen et al. (2003). For instance, using tasks involving the maintenance and manipulation of letter strings, Postle, Berger, \& D'Esposito (1999) found ventral and dorsal prefrontal and left SMG activations closely matching those observed in patient C.Z. during letter-by-letter reading. Those areas showed differential sensitivity to memory load and to the complexity of manipulations in working memory, pointing to distinct and interacting functional roles (see also Gruber \& von Cramon, 2003).

\subsection{Interhemispheric connections}

If the network subtending letter-by-letter reading is distributed across the two hemispheres, its operation requires spared interhemispheric connections, to ensure the transfer of letter identities from the RH to the LH. It has been proposed that in alexic patients with an additional callosal lesion, this transfer is impeded, yielding global alexia rather than letter-by-letter reading (Binder \& Mohr, 1992; Cohen et al., 2003). Therefore it seems surprising that patient C.Z. could read letter-by-letter despite the resection of her posterior callosal connections (Fig. 1). The case of patient C.Z. is indeed inconsistent with Binder and Mohr (1992) conclusion. These authors isolated the white matter above the posterior ventricular horn as the critical interhemispheric pathway allowing letter-by-letter reading, a region which had been removed in patient C.Z.

To gain some clarification on this issue, one may distinguish three types of interhemispheric communication involved in normal reading and in alexia. First, normal reading of letter displayed in the LVF is based on projections from right-sided visual cortex such as V4 toward the 
VWFA in the left fusiform gyrus. Lesion data and diffusion tensor imaging indicates that this pathway goes through the splenium of the corpus callosum, possibly its most ventral segment (Suzuki et al., 1998), and over the lateral ventricles (Molko et al., 2002). Severing those connections yields alexia in the LVF. Second, we propose that during letter-by-letter reading, the R-VWFA communicates the outcome of letter identification to the LH language system through a pathway whose anatomical substrate is not precisely defined. Severing those connections should prevent letter-by-letter reading in alexic patients. It is plausible that such connexions are more anterior than those projecting to the VWFA, as they connect more anterior regions. Such heterotopic callosal connections have been evidenced by Di Virgilio \& Clarke (1997), who studied the brain of a prosopagnosic patient with a right occipito-temporal lesion overlapping with the R-VWFA. They showed that monosynaptic projection from this region reach language areas in the LH, including Broca's area, Wernicke's area and the inferior parietal lobule. Such connections could be involved in the transfer of letter identities across hemispheres. However, their exact trajectory in the callosum and the hemispheric white matter is not known. Third, there are indications that semantic information can be transferred from the right to the left hemisphere through the anterior half of the corpus callosum. This pathway allows for the indirect naming of words displayed to the $\mathrm{RH}$, at least in subjects whose $\mathrm{RH}$ can access the meaning of printed words or numbers (Cohen \& Dehaene, 1996; Gazzaniga, 2000; Sidtis, Volpe, Holtzman, Wilson, \& Gazzaniga, 198). It is possible that the strategic transfer of letter identites across hemispheres during letter-by-letter reading can use a variety of more or less anterior anatomical pathways, and thus adapt to a variety of callosal lesions. Future research combining diffusion tensor and functional imaging may help delineate the anatomical substrates of those different types of interhemispheric exchange (Catani et al., 2003; Molko et al., 2002).

\subsection{Residual parallel letter perception?}

Although the focus of the present study was on letter-byletter reading, we conclude by briefly discussing the open issue of residual parallel letter processing in pure alexia. In the initial behavioural study, we observed that patient C.Z. performed quite well when asked to discriminate words from consonant strings, responding even faster than when reading aloud a single letter. There were some suggestions that the detection of consonant strings was based on parallel letter processing: Response latencies were short, independent of stimulus length, and the patient could detect consonant clusters located at the end of flashed words, i.e. beyond the scope of letter-by-letter reading. Indeed a number of studies are indicative of parallel perception in implicit reading tasks in pure alexic patients. Implicit reading has been documented using lexical decision, semantic decision, or word superiority effect in letter detection (for reviews see Arguin, Bub, \&
Bowers, 1998; Coslett \& Saffran, 1998). The performance of alexic patients in those tasks do not result from letter-byletter reading, a strategy which must even be carefully prevented to allow implicit reading to emerge (Coslett, Saffran EM, Greenbaum S, \& Schwartz, 1993). Accordingly, whenever word length was studied, no influence of this parameter on lexical decision was found (Bub \& Arguin, 1995; Coslett \& Saffran, 1989). Similarly, briefly displayed masked words, although they escaped explicit report, primed subsequent targets in an alexic patient. Importantly, the priming effect seemed to depend on the entire prime word, including its final letters (Bowers, Arguin, \& Bub, 1996). In brief, there are limited but consistent indications that implicit reading relies on parallel letter processing, as exemplified by patient C.Z.'s ability to detect consonant strings.

The cerebral mechanisms of such parallel perception in alexic patients is currently unknown. One hypothesis is that the intact RH visual system, presumably including the RVWFA, can identify several letters in parallel. However, the subsequent processing of those letters would depend on the current task demands (Cohen \& Dehaene, 1995; Coslett et al., 1993). When the only requirement is to pick out unfamiliar consonant strings, the RH could achieve the perceptual decision and trigger the response, with no need to convey to the LH the identity of individual letters. In contrast, when patients are engaged in explicit letter-by-letter reading, letters enter the internal spelling process only one at a time, maybe due to the attentional scanning of the stimulus, to the code used for the inter-hemispheric transfer of letter, to capacity limitations affecting this transfer, or to the incremental word recognition carried out by the LH. Those speculative hypotheses are compatible with the substantial network of activations observed in the patient's $\mathrm{RH}$, but should be assessed in the future with specifically tailored experimental designs.

\section{Acknowledgements}

We thank patient C.Z. for her participation. This study was supported by the IFR 49 and by a "Cognitique" grant from the French Ministry of Research.

\section{References}

Aghababian, V., \& Nazir, T. A. (2000). Developing normal reading skills: aspects of the visual processes underlying word recognition. Journal of Experimental Child Psychology, 76, 123-150.

Allison, T., McCarthy, G., Nobre, A., Puce, A., \& Belger, A. (1994). Human extrastriate visual cortex and the perception of faces words numbers and colors. Cerebral Cortex, 5, 544-554.

Allison, T., Puce, A., Spencer, D. D., \& McCarthy, G. (1999). Electrophysiological studies of human face perception. I: Potentials generated in occipitotemporal cortex by face and non-face stimuli. Cerebral Cortex, 9, 415-430.

Arguin, M., Bub, D. N., \& Bowers, J. S. (1998). Extent and limits of covert lexical activation in letter-by-letter reading. Cognitive Neuropsychology, 15, 53-92. 
Bartolomeo, P., Bachoud-Levi, A. C., Chokron, S., \& Degos, J. D. (2002). Visually- and motor-based knowledge of letters: evidence from a pure alexic patient. Neuropsychologia, 40, 1363-1371.

Bartolomeo, P., Bachoud-Levi, A. C., Degos, J. D., \& Boller, F. (1998). Disruption of residual reading capacity in a pure alexic patient after a mirror-image right-hemispheric lesion. Neurology, 50, 286-288.

Baynes, K., Eliassen, J. C. (1998). The visual lexicon: its access and organization in commissurotomy patients. In: M. Beeman, C., Chiarello (Eds.), Right hemisphere language comprehension (pp. 79-104). Mahwah, NJ: Lawrence Erlbaum Associates.

Beauregard, M., Chertkow, H., Bub, D., Murtha, S., Dixon, R., \& Evans, A. (1997). The neural substrate for concrete, abstract, and emotional word lexica: a positron emission tomography study. Journal of Cognitive Neuroscience, 9, 441-461.

Behrmann, M., Black, S. E., \& Bub, D. (1990). The evolution of pure alexia: a longitudinal study of recovery. Brain and Language, 39, 405427.

Behrmann, M., Plaut, D. C., \& Nelson, J. (1998). A literature review and new data supporting an interactive account of letter-by-letter reading. Cognitive Neuropsychology, 15, 7-51.

Beversdorf, D. Q., Ratcliffe, N. R., Rhodes, C. H., \& Reeves, A. G. (1997). Pure alexia: clinical-pathologic evidence for a lateralized visual language association cortex. Clinical Neuropathology, 16, 328-331.

Binder, J. R., \& Mohr, J. P. (1992). The topography of callosal reading pathways. A case-control analysis. Brain, 115, 1807-1826.

Booth, J. R., Burman, D. D., Meyer, J. R., Gitelman, D. R., Parrish, T. B., \& Mesulam, M. M. (2002). Functional anatomy of intra- and cross-modal lexical tasks. Neuroimage, 16, 7-22.

Booth, J. R., Burman, D. D., Meyer, J. R., Gitelman, D. R., Parrish, T. B., \& Mesulam, M. M. (2003). Relation between brain activation and lexical performance. Human Brain Mapping, 19, 155-169.

Bowers, J. S., Arguin, M., \& Bub, D. N. (1996). Fast and specific access to orthographic knowledge in a case of letter-by-letter surface alexia. Cognitive Neuropsychology, 13, 525-567.

Brett, M., Leff, A. P., Rorden, C., \& Ashburner, J. (2001). Spatial normalization of brain images with focal lesions using cost function masking. Neuroimage, 14, 486-500.

Brunswick, N., McCrory, E., Price, C. J., Frith, C. D., \& Frith, U. (1999). Explicit and implicit processing of words and pseudowords by adult developmental dyslexics: A search for Wernicke's Wortschatz. Brain, 122, 1901-1917.

Bub, D. N., \& Arguin, M. (1995). Visual word activation in pure alexia. Brain and Language, 49, 77-103.

Burton, M. W., Small, S. L., \& Blumstein, S. E. (2000). The role of segmentation in phonological processing: an fMRI investigation. Journal of Cognitive Neuroscience, 12, 679-690.

Cabeza, R., \& Nyberg, L. (2000). Imaging cognition II: an empirical review of 275 PET and fMRI studies. Journal of Cognitive Neuroscience, 12, 1-47.

Catani, M., Jones, D. K., Donato, R., \& Ffytche, D. H. (2003). Occipitotemporal connections in the human brain. Brain, 126, 2093-2107.

Chanoine, V., Teixeira Ferreira, C., Demonet, J. F., Nespoulous, J. L., \& Poncet, M. (1998). Optic aphasia with pure alexia: a mild form of visual associative agnosia? A case study. Cortex, 34, 437-448.

Cohen, L., \& Dehaene, S. (1995). Number processing in pure alexia: the effect of hemispheric asymmetries and task demands. NeuroCase, 1 , 121-137.

Cohen, L., \& Dehaene, S. (1996). Cerebral networks for number processing: evidence from a case of posterior callosal lesion. NeuroCase, 2, 155-174.

Cohen, L., Dehaene, S., Naccache, L., Lehéricy, S., Dehaene-Lambertz, G., \& Hénaff, M. A. et al., (2000). The visual word form area: spatial and temporal characterization of an initial stage of reading in normal subjects and posterior split-brain patients. Brain, 123, 291-307.

Cohen, L., Lehericy, S., Chochon, F., Lemer, C., Rivaud, S., \& Dehaene, S. (2002). Language-specific tuning of visual cortex? Functional properties of the Visual Word Form Area. Brain, 125, 1054-1069.
Cohen, L., Martinaud, O., Lemer, C., Lehericy, S., Samson, Y., \& Obadia, M. et al., (2003). Visual word recognition in the left and right hemispheres: anatomical and functional correlates of peripheral alexias. Cerebral Cortex, 13, 1313-1333.

Coltheart, M. (1980). Deep dyslexia: a right-hemisphere hypothesis. In: M. Coltheart, K. Patterson, J. C. Marshall (Eds.), Deep dyslexia (pp. 326-380). London: Routledge.

Coslett, H. B., \& Monsul, N. (1994). Reading with the right hemisphere: evidence from transcranial magnetic stimulation. Brain and Language, 46, 198-211.

Coslett, H. B., \& Saffran, E. M. (1989). Evidence for preserved reading in pure alexia. Brain, 112, 327-359.

Coslett, H., Saffran, E. (1998). Reading and the right hemisphere: eidence from acquired dyslexia. In: M., Beeman, C., Chiarello (Eds.), Right hemisphere language comprehension ( pp. 105-132). Mahwah, NJ: Lawrence Erlbaum Associates.

Coslett, H. B., Saffran, E. M., Greenbaum, S., \& Schwartz, H. (1993). Reading in pure alexia: the effect of strategy. Brain, 116, 21-37.

Damasio, A. R., \& Damasio, H. (1983). The anatomic basis of pure alexia. Neurology, 33, 1573-1583.

Dehaene, S., Jobert, A., Naccache, L., Ciuciu, P., Poline, J. B., Le Bihan, D., \& Cohen, L. (2004). Letter binding and invariant recognition of masked words. Psychological Science, 15, 307-313.

Dehaene, S., Le Clec'H, G., Poline, J. B., Le Bihan, D., \& Cohen, L. (2002). The visual word form area: a prelexical representation of visual words in the fusiform gyrus. Neuroreport, 13, 321-325.

Dehaene, S., Naccache, L., Cohen, L., Bihan, D. L., Mangin, J. F., \& Poline, J. B. et al., (2001). Cerebral mechanisms of word masking and unconscious repetition priming. Nature Neuroscience, 4, 752-758.

Dejerine, J. (1892). Contribution à l'étude anatomo-pathologique et clinique des différentes variétés de cécité verbale. Mémoires de la Société de Biologie, 4, 61-90.

Dejerine, J. (1895). Anatomie des centres nerveux (vol 1). Paris: Rueff et Cie.

D'Esposito, M., Postle, B. R., \& Rypma, B. (2000). Prefrontal cortical contributions to working memory: evidence from event-related fMRI studies. Experimental Brain Research, 133, 3-11.

Di Virgilio, G., \& Clarke, S. (1997). Direct interhemispheric visual input to human speech areas. Human Brain Mapping, 5, 347-354.

Farah, M. J., \& Wallace, M. A. (1991). Pure alexia as a visual impairment: a reconsideration. Cognitive Neuropsychology, 8, 313-334.

Fiez, J. A., Balota, D. A., Raichle, M. E., \& Petersen, S. E. (1999). Effects of lexicality, frequency, and spelling-to-sound consistency on the functional anatomy of reading. Neuron, 24, 205-218.

Gauthier, I., Tarr, M. J., Moylan, J., Skudlarski, P., Gore, J. C., \& Anderson, A. W. (2000). The fusiform face area is part of a network that processes faces at the individual level. Journal of Cognitive Neuroscience, 12, 495-504.

Gazzaniga, M. S. (2000). Cerebral specialization and interhemispheric communication. Does the corpus callosum enable the human condition. Brain, 123, 1293-1326.

Greenwald, M. L., \& Gonzalez Rothi, L. J. (1998). Lexical access via letter naming in a profoundly alexic and anomic patient: a treatment study. Journal of the International Neuropsychological Society, 4, 595-607.

Gruber, O., \& von Cramon, D. Y. (2003). The functional neuroanatomy of human working memory revisited. Evidence from 3-T fMRI studies using classical domain-specific interference tasks. Neuroimage, 19, 797-809.

Hasson, U., Harel, M., Levy, I., \& Malach, R. (2003). Large-scale mirror-symmetry organization of human occipito-temporal object areas. Neuron, 37, 1027-1041.

Hasson, U., Levy, I., Behrmann, M., Hendler, T., \& Malach, R. (2002). Eccentricity bias as an organizing principle for human high-order object areas. Neuron, 34, 479-490.

Kastner, S., De Weerd, P., Desimone, R., \& Ungerleider, L. G. (1998). Mechanisms of directed attention in the human extrastriate cortex as revealed by functional MRI. Science, 282, 108-111. 
Koutstaal, W., Wagner, A. D., Rotte, M., Maril, A., Buckner, R. L., \& Schacter, D. L. (2001). Perceptual specificity in visual object priming: functional magnetic resonance imaging evidence for a laterality difference in fusiform cortex. Neuropsychologia, 39, 184-199.

Lavidor, M., Ellis, A. W., Shillcock, R., \& Bland, T. (2001). Evaluating a split processing model of visual word recognition: effects of word length. Brain Research Cognitive Brain Research, 12, 265-272.

McCandliss, B. D., Cohen, L., \& Dehaene, S. (2003). The visual word form area: expertise for reading in the fusiform gyrus. Trends in Cognitive Science, 7, 293-299.

Miozzo, M., \& Caramazza, A. (1998). Varieties of pure alexia: The case of failure to access graphemic representations. Cognitive Neuropsychology, 15, 203-238.

Molko, N., Cohen, L., Mangin, J. F., Chochon, F., Lehéricy, S., \& Le Bihan, D. et al., (2002). Visualizing the neural bases of a disconnection syndrome with diffusion tensor imaging. Journal of Cognitive Neuroscience, 14, 629-636.

Montant, M., \& Behrmann, M. (2000). Pure alexia. Neurocase, 6, 265294.

Morais, J., \& Kolinsky, R. (1994). Perception and awareness in phonological processing: the case of the phoneme. Cognition, 50, 287297.

Muller, N. G., Bartelt, O. A., Donner, T. H., Villringer, A., \& Brandt, S. A. (2003). A physiological correlate of the Zoom Lens of visual attention. Journal of Neuroscience, 23, 3561-3565.

Nobre, A. C., Allison, T., \& McCarthy, G. (1994). Word recognition in the human inferior temporal lobe. Nature, 372, 260-263.

O'Craven, K. M., \& Kanwisher, N. (2000). Mental imagery of faces and places activates corresponding stimulus-specific brain regions. Journal of Neuroscience, 12, 1013-1023.

Oldfield, R. C. (1971). The assessment and analysis of handedness: the Edinburgh inventory. Neuropsychologia, 9, 97-113.

Patterson, K., Vargha-Khadem, F., \& Polkey, C. E. (1989). Reading with one hemisphere. Brain, 112, 39-63.

Paulesu, E., McCrory, E., Fazio, F., Menoncello, L., Brunswick, N., \& Cappa, S. F. et al., (2000). A cultural effect on brain function. Nature Neuroscience, 3, 91-96.

Pessoa, L., Kastner, S., \& Ungerleider, L. G. (2003). Neuroimaging studies of attention: from modulation of sensory processing to topdown control. Journal of Neuroscience, 23, 3990-3998.

Polk, T. A., \& Farah, M. J. (2002). Functional MRI evidence for an abstract, not perceptual, word-form area. Journal of Experimental Psychology General, 131, 65-72.

Postle, B. R., Berger, J. S., \& D’Esposito, M. (1999). Functional neuroanatomical double dissociation of mnemonic and executive control processes contributing to working memory performance. Proceedings of the National Academy of Sciences of United States of America, 96, 12959-12964.
Price, C. J., Wise, R. J. S., \& Frackowiak, R. S. J. (1996). Demonstrating the implicit processing of visually presented words and pseudowords. Cerebral Cortex, 6, 62-70.

Puce, A., Allison, T., Asgari, M., Gore, J. C., \& McCarthy, G. (1996). Differential sensitivity of human visual cortex to faces letterstrings, and textures: a functional magnetic resonance imaging study. Journal of Neuroscience, 16, 5205-5215.

Salmelin, R., Service, E., Kiesilä, P., Uutela, K., \& Salonen, O. (1996). Impaired visual word processing in dyslexia revealed with magnetoencephalography. Annals of Neurology, 40, 157-162.

Sidtis, J. J., Volpe, B. T., Holtzman, J. D., Wilson, D. H., \& Gazzaniga, M. S. (1981). Cognitive interaction after staged callosal section: evidence for transfer of semantic activation. Science, 212, 344-346.

Simos, P. G., Breier, J. I., Fletcher, J. M., Foorman, B. R., Castillo, E. M., \& Papanicolaou, A. C. (2002). Brain mechanisms for reading words and pseudowords: an integrated approach. Cerebral Cortex, 12, 297-305.

Somers, D. C., Dale, A. M., Seiffert, A. E., \& Tootell, R. B. H. (1999). Functional MRI reveals spatially specific attentional modulation in human primary visual cortex. Proceedings of the National Academy of Sciences of United States of America, 96, 1663-1668.

Speedie, L. J., Rothi, L. J., \& Heilman, K. M. (1982). Spelling dyslexia: a form of cross-cuing. Brain Lang, 15, 340-352.

Super, H., Spekreijse, H., \& Lamme, V. A. (2001). A neural correlate of working memory in the monkey primary visual cortex. Science, 293, $120-124$.

Suzuki, K., Yamadori, A., Endo, K., Fujii, T., Ezura, M., \& Takahashi, A. (1998). Dissociation of letter and picture naming resulting from callosal disconnection. Neurology, 51, 1390-1394.

Talairach, J., Tournoux, P. (1988). Co-planar stereotaxic-atlas of the human brain. 3-Dimensional proportional system: approach to cerebral imaging. Translated by Mark Rayport. New-York: Thieme Medical Publishers Inc.

Tarkiainen, A., Helenius, P., Hansen, P. C., Cornelissen, P. L., \& Salmelin, R. (1999). Dynamics of letter string perception in the human occipitotemporal cortex. Brain, 122, 2119-2132.

Vuilleumier, P., Sagiv, N., Hazeltine, E., Poldrack, R. A., Swick, D., \& Rafal, R. D. et al., (2001). Neural fate of seen and unseen faces in visuospatial neglect: a combined event-related functional MRI and event-related potential study. Proceedings of the National Academy of Sciences of United States of America, 98, 3495-3500.

Wagner, A. D., Schacter, D. L., Rotte, M., Koutstaal, W., Maril, A., \& Dale, A. M. et al., (1998). Building memories: remembering and forgetting of verbal experiences as predicted by brain activity. Science, $281,1188-1191$.

Weekes, B. S. (1997). Differential effects of number of letters on word and nonword naming latency. Quarterly Journal of Experimental Psychology, 50A, 439-456. 